\title{
Identification of the Nuclear Localization Signal Region of Duck Enteritis Virus UL14 and Its Interaction with VP16
}

\author{
FangJie Li ${ }^{\mathrm{a}-\mathrm{c}}$ YuWei Zhang ${ }^{\mathrm{a}-\mathrm{c}}$ Shun Chen ${ }^{\mathrm{a}-\mathrm{c}}$ MingShu Wang ${ }^{\mathrm{a}-\mathrm{c}}$ \\ RenYong Jia ${ }^{\mathrm{a}-\mathrm{c}}$ DeKang Zhu ${ }^{\mathrm{a}-\mathrm{c}}$ MaFeng Liu ${ }^{\mathrm{a}-\mathrm{c}} \quad K_{\text {KunFeng Sun }}^{\mathrm{a}-\mathrm{c}}$ \\ Qiao Yang ${ }^{\mathrm{a}-\mathrm{c}}$ Ying Wu ${ }^{\mathrm{a}-\mathrm{c}}$ XiaoYue Chen ${ }^{\mathrm{b}}$ AnChun Cheng ${ }^{\mathrm{a}-\mathrm{c}}$ \\ ${ }^{a}$ Institute of Preventive Veterinary Medicine, Sichuan Agricultural University, ${ }^{b}$ Avian Disease Research Center, \\ College of Veterinary Medicine of Sichuan Agricultural University, and ${ }^{\mathrm{C}}$ Key Laboratory of Animal Disease and \\ Human Health of Sichuan Province, Sichuan Agricultural University, Chengdu, PR China
}

\section{Keywords}

Duck enteritis virus · pUL14 · VP16 · Interaction .

Nonclassical nuclear localization signal

\begin{abstract}
Object: Duck enteritis virus (DEV) is a member of the Alphaherpesvirinae viruses. VP16 and pUL14 are both predicted to be tegument proteins of DEV. Methods: An indirect immunofluorescence assay (IFA) was performed for preliminary analysis of the colocalization of pUL14 and VP16, which detected their subcellular localization in duck embryo fibroblasts (DEFs) during virus replication. The coexpression of pUL14 and VP16 was detected in transfected DEFs. A bimolecular fluorescence complementation (BiFC) assay was used to confirm a direct interaction between pUL14 and VP16. To better characterize the nuclear localization domain of pUL14, we designed a series of deletion mutants and transfected them with VP16. Results: Our IFA findings indicated that pUL14 binds to VP16 in the cytoplasm and that pUL14 leads VP16 import into the nucleus during DEV replication. The BiFC assay revealed the presence of pUL14 and VP16 complexes. Furthermore, 1-98 amino acid (aa) at the $\mathrm{N}$-terminus of pUL14 played a role in the nuclear localization signal (NLS)
\end{abstract}

region and promoted translocation of VP16 into the nucleus to complete the virus life cycle. Conclusions: Our findings indicated that pUL14 could transport VP16 into the nucleus and that the N-terminal 1-98 aa may contain the NLS domain of pUL14.

(c) 2017 S. Karger AG, Basel

\section{Introduction}

Duck enteritis virus (DEV) can infect up to $100 \%$ of populations of birds, such as ducks, geese, and wild waterfowl, in the order Anseriformes and can cause an acute infectious disease $[1,2]$. A high prevalence of DEV was found among free-ranging water birds in Poland [3]. The economic impact on waterfowl husbandry caused by $\mathrm{DEV}$ is considerable because the mortality, especially in young birds, can reach $100 \%[2,3]$. As a member of Alphaherpesvirinae, DEV contains 4 distinct structures: the core, capsid, tegument, and envelope $[4,5]$. The tegument

FangJie Li and YuWei Zhang contributed equally to this work and should be considered as first authors.

\section{KARGER}

E-Mail karger@karger.com www.karger.com/int
(C) 2017 S. Karger AG, Basel

0300-5526/17/0594-0187\$39.50/0
AnChun Cheng

Sichuan Agricultural University

Chengdu 610000 (China)

E-Mail chenganchun@vip.163.com 
Table 1. Primers used for the UL14 gene deletion mutants

\begin{tabular}{llc}
\hline Recombinant vectors & Primers & Fragment limits, aa \\
\hline pEGFPN1-m1 & F: AAGCTTAACATGCTATCGCGGCTTCAAGATC & $1-45$ \\
pEGFPN1-m2 & F: GGATCCGTGTCAACGAACACGGCACTATT & \\
pEGFPN1-m3 & R: GGATTCATGACTTTTACGTCAGCGAAAGC & $46-98$ \\
pEGFPN1-m4 & F: AAGCTTAACATGCTATCGCGGCTTCAAGATC & $1-98$ \\
& R: GGATCCGATAAAAACCTTCTGTGCGCC & \\
& F: AAGCTTATGTCGCCAGGCCTAATTGAT & $99-152$ \\
& R: GGATCCGCTAATAGGGCTTCGTCGTCTTC & \\
\hline
\end{tabular}

The HindIII and BamHI restriction sites are underlined.

is not highly structured and is distributed around the capsid [6]. It contributes to viral replication and immune evasion $[6,7]$. Tegument assembly into a virion occurs via a series of processes that entails numerous interactions, including tegument-capsid, tegument-envelope, and tegument-tegument [8-13]. The tegument-tegument interactions constitute a dense network of associations among tegument proteins [8].

In DEV, the protein UL14 (pUL14) was predicted to be a minor component of the virion tegument protein, which is not essential for viral replication, and VP16 (UL48) was predicted to be a large tegument protein that is essential for viral assembly [14]. Although there is little information available about pUL14 and VP16 in DEV, they have been better characterized in herpes simplex virus type 1 (HSV-1) and HSV-2. The pUL14 has been shown to interact with tegument protein VP16 and capsid protein VP26 $[15,16]$. Additionally, pUL14 may be required for efficient envelopment when particles fuse into cytoplasmic vesicles, promoting the spread of HSV-1 infection [17]. Alternatively, it has been reported that VP16 can interact with many viral tegument proteins and the cytoplasmic domains of glycoprotein, which indicates that VP16 has many potential interactions during virus infection $[12,13$, 18-20]. By contrast, there is little information available about interactions between the 2 tegument proteins pUL14 and VP16 in DEV. The present study was designed to gain insight into the assembly of tegument protein pUL14 and VP16 during DEV infection.

A viral protein localized to the nucleus may contribute to host cell proliferation, development, apoptosis, and reactions [21, 22]. It has been determined that the proteins localized to the nucleus carry nuclear localization signals (NLS) [23]. There are 3 types of NLS, including classical NLS that contains a single peptide of basic amino acids, bipartite NLS that consists of 2 stretches of basic amino acids that are separated by a linker region, and nonclassical NLS that has no obvious features of a basic amino acid signal sequence [24-29]. Although various NLS can differ in structure, they can perform the same function depending on protein recognition by soluble factors that mediate transport via the nuclear pore complexes [30]. To detect a presumptive NLS, a commonly used method is to assess the distribution of GFP [31]. There were no classical NLS in DEV pUL14, which was analyzed using the NLS prediction program of the PSORT II server. However, there were 4 a-helical segments in the 1-152 amino acid (aa) sequence of pUL14: 4-41, 55-103, 107-133, and 146-152 aa [32]. Subsequently, we studied the function of the secondary structure of DEV UL14 in nuclear import mediated by 1 of the $4 a$-helical segments, which showed the same activity as nonclassical NLS. Immunofluorescence assay (IFA), coexpression, and bimolecular fluorescence complementation (BiFC) assessments were performed to gain insight into the assembly of tegument proteins pUL14 and VP16 during DEV infection. To determine the function of nonclassical pUL14 and its association with VP16, we constructed a series of UL14 mutants. This study will shed light on the molecular pathogenic mechanism of DEV.

\section{Materials and Methods}

Viruses and Cells

DEV was prepared at the Key Laboratory of Animal Disease and Human Health of Sichuan Province at Sichuan Agricultural University according to a method that has been previously reported. Duck embryo fibroblasts (DEFs) were cultured in Minimum Essential Medium (Gibco-BRL) with 10\% fetal bovine serum (FBS). Then, cells were maintained in 2\% FBS and incubated in 5\% $\mathrm{CO}_{2}$ and $37^{\circ} \mathrm{C}$ as previously reported for our laboratory [33]. 


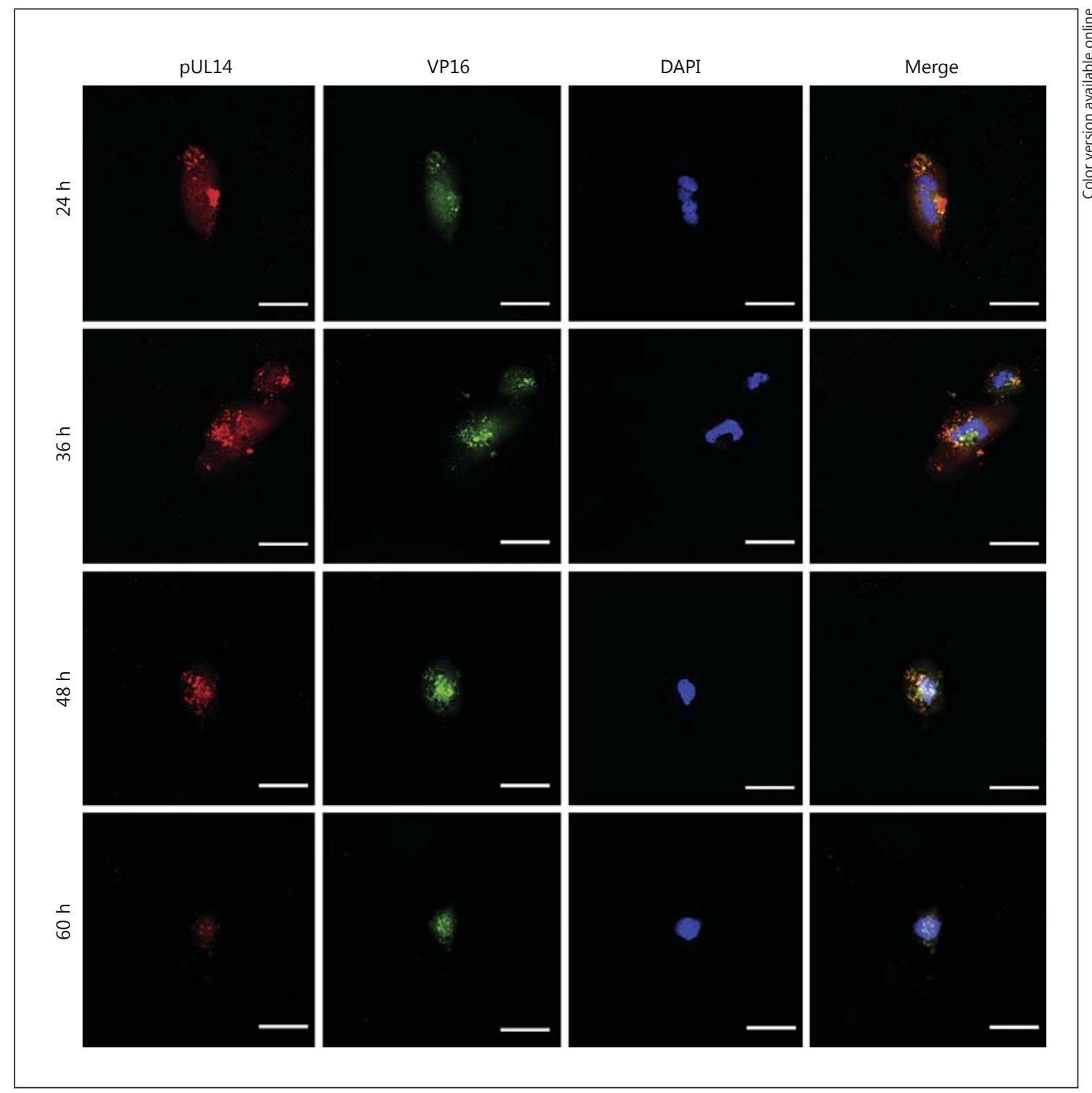

Fig. 1. Intracellular localization of pUL14 and VP16 in DEV-infected cells. Cells were infected with DEV at a MOI of $2 \mathrm{PFU} /$ cell and were fixed at 24,36, 48, and 60 h.p.i. Cells were incubated with rabbit anti-VP16 primary antibody (PAb) and mouse anti-pUL14 PAb primary antibodies, and Alexa Fluor 594-conjugated goat anti-mouse and Alexa Fluor 488-conjugated goat anti-rabbit secondary antibodies. The subcellular localization of these proteins was assessed by visualizing the fluorescence. Scale bars, $10 \mu \mathrm{m}$.

Plasmid Construction

To detect interactions between pUL14 and VP16, we constructed the plasmids pBiFCVC155-UL14 and pBiFCVN155-UL48. The primers for pBiFC-VC155-UL14 were F: GTCGACCATGCTATCGCGGCTTCAAGATC and R: GGTACCGCTAATAGGGCTTCGTCGTCTTC, with SalI and KpnI restriction sites, respectively. The primers for pBiFCVN155-UL48 were F: GTCGACTGTTCGTATTATTGGGTA and R: GGTACCTATGTAGGCTAATTATCTGGCGAG, with SalI and KpnI restriction sites, respectively.

Identification of the NLS Region of DEV UL14 and Its Interaction with VP16
To assess the colocalization of pUL14 and VP16, the eukaryotic expression plasmids pEGFP-UL14 and pDsRed-UL48 were constructed. The primers for pEGFP-UL14 were F: AAGCTTAACATGCTATCGCGGCTTCAAGATC and R: GGATCCGCTAATAGGGCTTCGTCGTCTTC, with HindIII and BamHI restriction sites, respectively. The primers for pDsRed-UL48 were F: GAGCTCATTATTGGGTAAATAGGTGTGGGTG and R: CCGCGGATTGGATCTCGTGATTAGTCACATG, with SacI and SacII restriction sites, respectively. Primers were designed according to sequences available in GenBank with accession No. JQ647509 
Fig. 2. Subcellular localization of pUL14 and VP16. Cells were fixed at $48 \mathrm{~h}$ postcotransfection. The pEGFP-UL14 alone (green fluorescence) and pDsRed-UL48 (red fluorescence) plasmids were used (colors refer to the online version only). Cells were transfected or cotransfected with pEGFP-UL14 and pDsRed-UL48. Scale bars, $10 \mu \mathrm{m}$. YFP, yellow fluorescent protein.

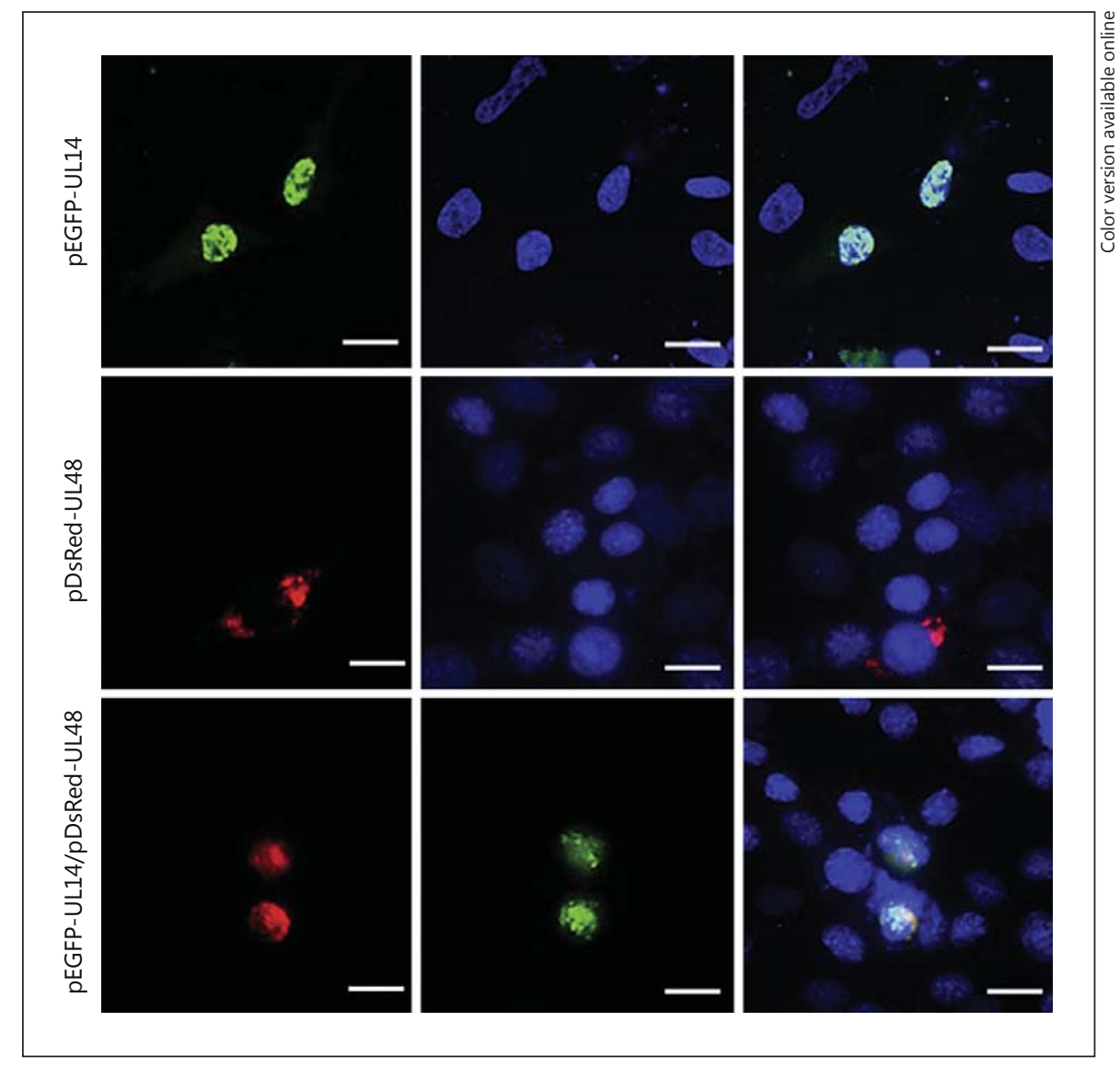

[34]. The DEV genome was used as a PCR template. PCR products were sequenced (Invitrogen) and cloned into eukaryotic vectors according to the instructions for T4 DNA ligase (Takara).

To identify the nonclassical NLS of pUL14, we designed deletion mutants of the UL14 gene. The secondary structure of pUL14 was analyzed using a protein prediction server (https://www.predictprotein.org) and $4 a$-helixes were predicted [32]. To analyze these helixes in the nuclear targeting function, a series of deletion mutants were designed. Primers for the deletion mutant plasmids pEGFPN1$\mathrm{m} 1$ (m1), pEGFPN1-m2 (m2), pEGFPN1-m3 (m3), and pEGFPN1$\mathrm{m} 4$ (m4) were designed and contained HindIII and BamHI restriction sites (Table 1). PCR products were ligated into pEGFP-N1, and all constructs were verified by sequencing (Invitrogen).

\section{Indirect Immunofluorescence Assays}

To examine the subcellular localization of pUL14 and VP16 during virus infection, an indirect IFA was used as previously reported [35]. First, DEV was used to infect DEFs that were grown on coverslips. Then, we harvested coverslips at $24,36,48$, and $60 \mathrm{~h}$ post infection (h.p.i.) and fixed them with $4 \%$ formaldehyde for $30 \mathrm{~min}$ at $4^{\circ} \mathrm{C}$. Cells were then permeabilized and blocked. The cells were incubated with mouse anti-UL14 IgG and rabbit antiVP16 primary antibodies at $4^{\circ} \mathrm{C}$. Then, we incubated them with Alexa Fluor 594-conjugated goat anti-mouse and Alexa Fluor 488-conjugated goat anti-rabbit secondary antibodies (Bio-Rad). Cell nuclei were stained using DAPI $(5 \mu \mathrm{g} / \mathrm{mL}$, Beyotime). Finally, florescent images were viewed and recorded using a Nikon 80i fluorescence microscope (Nikon Inc., Kanagawa, Japan).

\section{Transfection and Transient Expression}

DEFs were grown on coverslips with $10 \%$ FBS (Gibco). After $24 \mathrm{~h}$, DEFs were transfected with pBiFCVC155-UL14, pBiFCVN155-UL48, pEGFPN1-UL14, and pDsRed-UL48, according to the manufacturer's instructions for Lipofectamine 3000 (Invitrogen). After $48 \mathrm{~h}$, cells were fixed, permeabilized, and stained with DAPI. Finally, florescence was visualized with a Nikon 80 i fluorescence microscope [36].

To determine the nonclassical NLS of pUL14, deletion mutants (m1-m4) were transfected into DEFs. To analyze the transport function of the nonclassical NLS, deletion mutants of pUL14 and pDsRed-UL48 were cotransfected into DEFs. Cells on slides were treated as described above.

\section{Results}

\section{Colocalization of pUL14 and VP16 by IFA}

To detect the subcellular localization of pUL14 and VP16 during DEV replication in DEFs, IFA was performed. Cells were fixed at 24, 36, 48, and 60 h.p.i. Cells 
Fig. 3. The BiFC assay was used to detect interactions between pUL14 and VP16. Cells were fixed and examined by fluorescence microscopy to confirm transfection at $48 \mathrm{~h}$ posttransfection. No fluorescence was detected in the pBiFCVN155-UL48/ pBiFCVC155 and pBiFCVC155-UL14/ pBiFCVN155 plasmid groups. By contrast, fluorescence was detected in the $\mathrm{pBiF}$ CVN155-UL48/pBiFCVC155-UL14 group. Bars, $10 \mu \mathrm{m}$. YFP, yellow fluorescent protein.

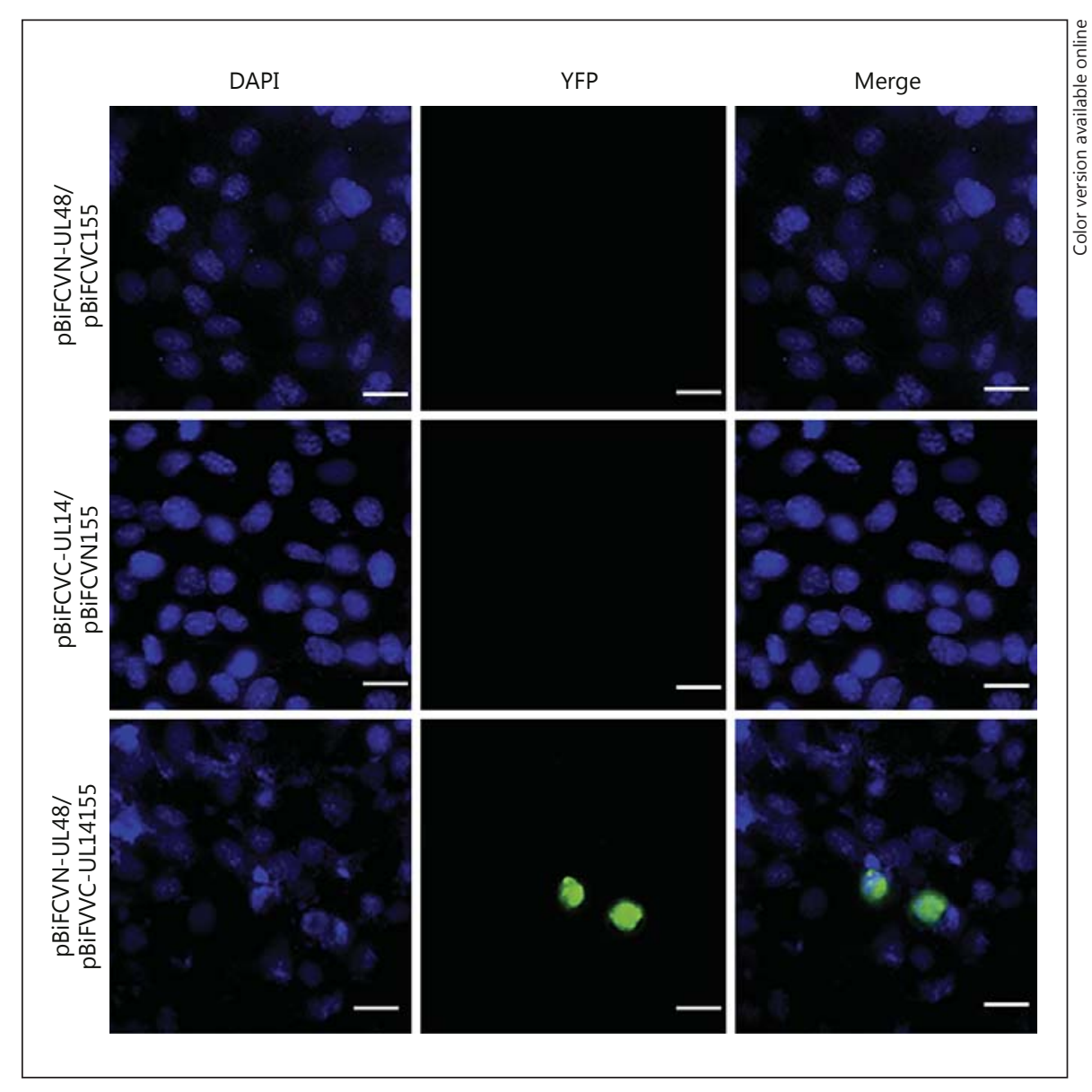

were incubated with rabbit anti-VP16 and mouse antipUL14 primary antibody, followed by Alexa Fluor 594-conjugated goat anti-mouse and Alexa Fluor 488-conjugated goat anti-rabbit secondary antibodies. Both red and greed fluorescence could be detected in the cytoplasm at 24 h.p.i. Subsequently, fluorescence could be observed in the perinuclear region at 36 h.p.i. The fluorescence was both in the perinuclear region and nucleus at 48 h.p.i. Both red and green fluorescence could be observed in the nucleus at 60 h.p.i. (Fig. 1). Although the colocalization was not obvious from $24-36$ h.p.i., it was more robust at 60 h.p.i.

\section{pUL14 Is Involved in VP16 Nuclear Transport}

As we observed colocalization of pUL14 and VP16 during infection, we decided detect the subcellular localization of pUL14 and VP16 by overexpressing the 2 proteins. The DEFs were transfected with 3 groups of plasmids: pEGFP-UL14, pDsRed-UL48, and pEGFP-UL14/ pDsRed-UL48. After $48 \mathrm{~h}$, pUL14 was detected in the nucleus by detecting green fluorescence, where pEGFPUL14 was transcribed into DEFs alone. VP16 was detected in the cytoplasm by detecting red fluorescence, where pDsRed-UL48 was transcribed into DEF alone. In group 3, plasmids pEGFP-UL14 and pDsRed-UL48 were cotransfected, and the subcellular localization of pUL14 and VP16 were both nuclear (Fig. 2).

\section{Confirmation of Interactions between pUL14 and VP16 by BiFC}

To determine whether there was a direct interaction between pUL14 and VP16, we performed BiFC assays. DEFs grown on coverslips that were transfected with plasmids divided into 3 groups: pBiFCVN155-UL48/ pBiFCVC155, pBiFCVC155-UL14/pBiFCVN155, and pBiFCVN155-UL48/pBiFCVC155-UL14. We found that there was no fluorescence in the pBiFCVN155UL48/pBiFCVC155 group and pBiFCVC155-UL14/ pBiFCVN155 group, whereas fluorescence was detected in the pBiFCVN155-UL48/pBiFCVC155-UL14 group 


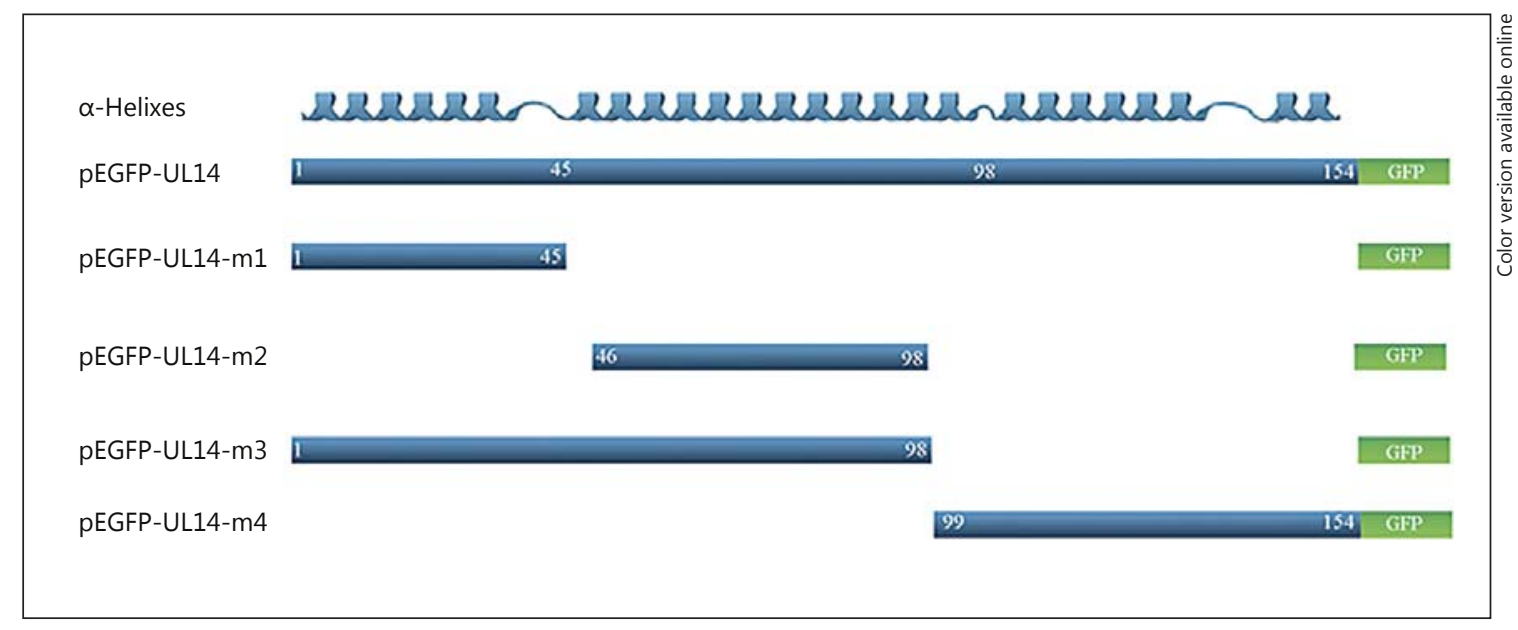

Fig. 4. The structures of the pUL14 deletion mutants. The $4 \alpha$-helixes of pUL14 were predicted and are represented as blue helixes. According to the helixes, pUL14 mutants were designed. Blue strips (color in online version only) show the different regions of pUL14 and green strips show the GFP regions.

(Fig. 3). This finding suggested that green fluorescence was produced by an interaction between pUL14 and VP16 (Fig. 3).

\section{Intracellular Localization of the UL14 Deletion \\ Mutants}

To identify the nuclear localization domain of pUL14, we designed a series of deletion mutants. The secondary structure of pUL14 was analyzed and revealed the presence of 4 a-helixes (Fig. 4). DEFs were transfected with $\mathrm{m} 1-\mathrm{m} 4$ and pEGFPN1. We detected the localization of the pUL14 mutants via GFP fluorescence localization (Fig. 5). These findings indicated that the GFP fluorescence of UL14-m1 (1-45 aa), UL14-m2 (46-98 aa), and UL14-m4 (99-152 aa) were each diffuse, both in the nucleus and cytoplasm. UL14-m3 (1-98 aa) was distributed in the nucleus.

The Region 1-98 a of pUL14 Has an NLS for VP16

As pUL14 could transport VP16 into the nucleus, we further confirmed that the NLS domain of pUL14 played a role in transporting VP16. UL14-m1, UL14-m2, UL14$\mathrm{m} 3$, and UL14-m4 were coexpressed with VP16 in DEFs to determine the nuclear transport function of the nonclassical NLS in pUL14. Colocalization of $\mathrm{m} 3$ and VP16 was observed. No colocalization of the 2 proteins in the nucleus was detected in the other groups (Fig. 6). This finding confirmed that the 1-98 aa at the N-terminus of pUL14 plays a key role in the nuclear localization of VP16.

\section{Discussion}

The BiFC assay has been widely used to detect interactions between 2 proteins [37-39]. In this study, BiFC assays were used to determine whether there was an interaction between pUL14 and VP16. Expression plasmids that encoded pUL14 or VP16 fused to a C- or N-terminal fragment of yellow fluorescent protein were generated and transfected into DEFs. The 2 nonfluorescent fragments of yellow fluorescent protein could associate and form a fluorescent complex by the fusion of 2 proteins that interact with each other [40]. Our findings revealed that 2 fragments of yellow fluorescent protein interacted, as they produced fluorescence. In this study, the results of the BiFC assay suggested that interactions occur between pUL14 and VP16.

As DEV pUL14 localized to the nucleus, we sought to identify the nonclassical NLS of DEV pUL14. As it had 4 helixes, we designed helix deletion mutants and then assessed the helix distribution in transfected DEFs. GFP distribution was used as a criterion to detect the presump-

Fig. 5. Schematic diagram of plasmids encoding mutants of DEV pUL14 fused with GFP. Subcellular localization of the DEV UL14 mutants in DEF cells transfected using Lipofectamine 3000, including pEGFP-UL14-m1 (m1), pEGFP-UL14-m2 (m2), pEGFPUL14-m3 (m3), pEGFP-UL14-m4 (m4), and pEGFP-N1. GFP fluorescence of UL14-m1 (1-45 aa), UL14-m2 (46-98 aa), and UL14m4 (99-152 aa) revealed diffuse staining in cells. UL14-m3 (1-98 aa) showed a nuclear distribution. Scale bars, $10 \mu \mathrm{m}$.

(For figure see next page.)

Li/Zhang/Chen/Wang/Jia/Zhu/Liu/Sun/ Yang/Wu/Chen/Cheng 


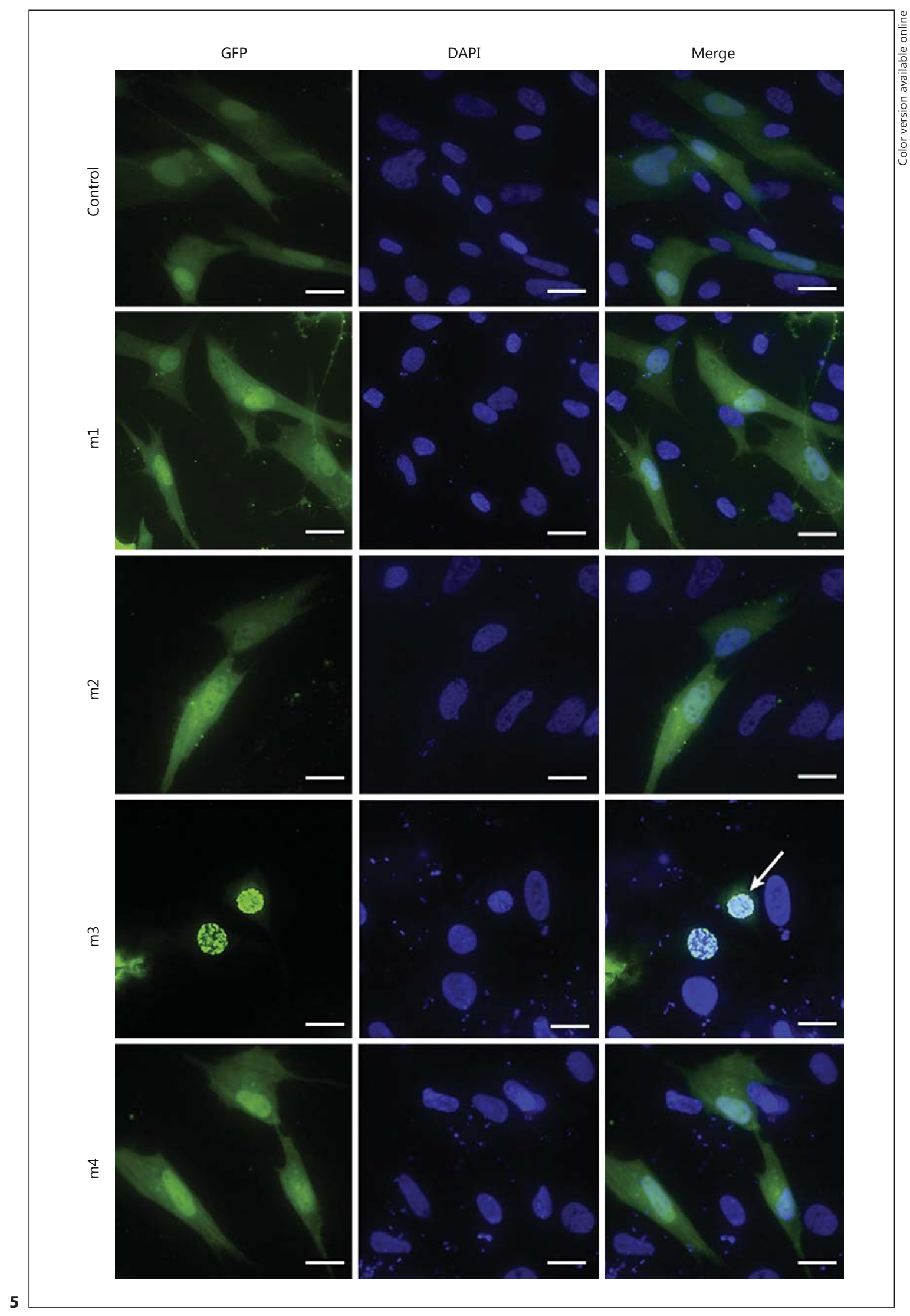




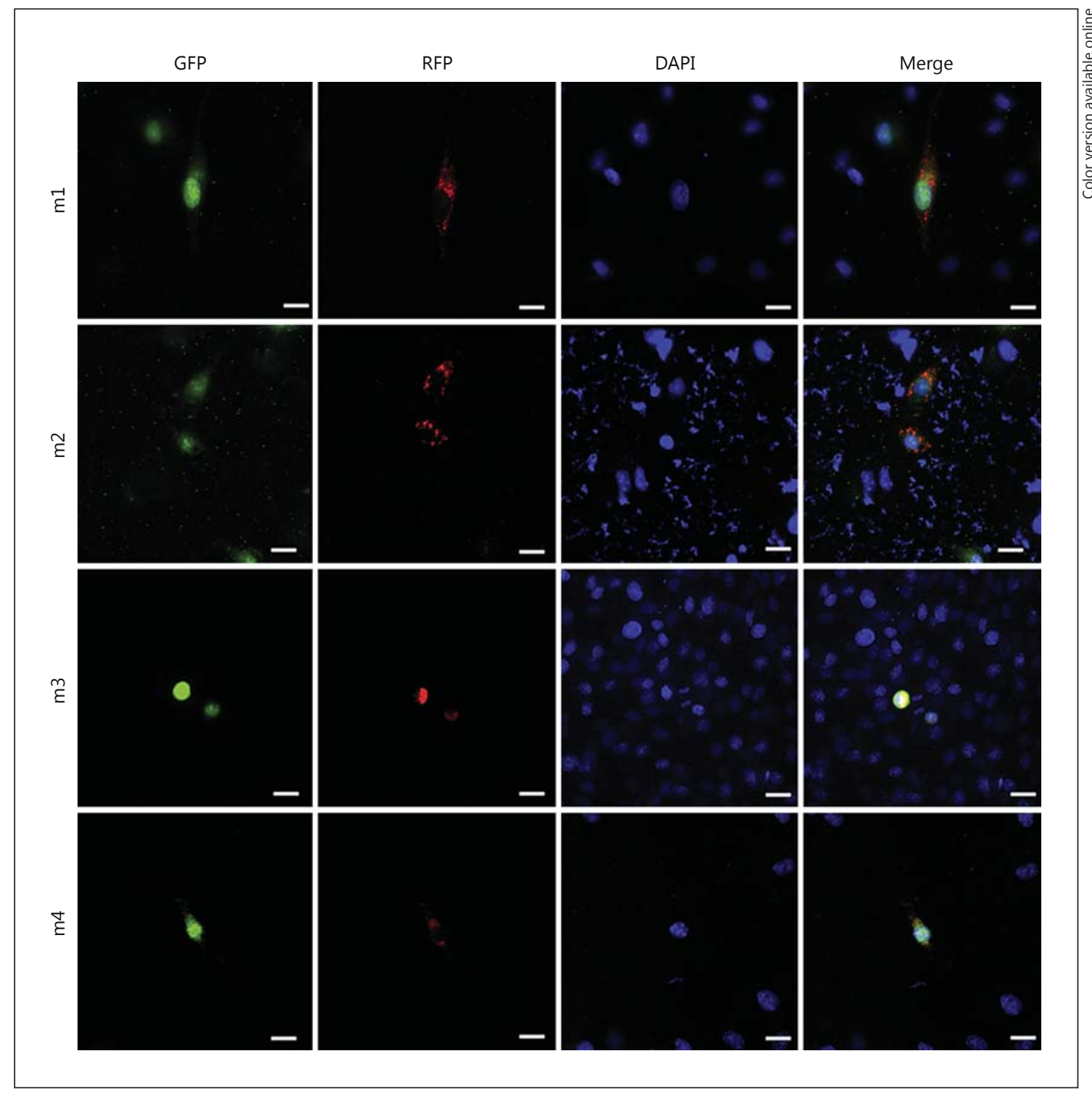

Fig. 6. Subcellular localization of VP16 deletion mutants. Cells were fixed at $48 \mathrm{~h}$ post-cotransfection. Scale bars, $10 \mu \mathrm{m}$.

tive nonclassical NLS of UL14. As previously reported, the molecular mass of GFP is $27 \mathrm{kDa}$ and it can freely diffuse in the nucleus and cytoplasm [41, 42]. Our results indicated that the mutants, including UL14-m1 (1-45 aa), UL14-m2 (46-98 aa), and UL14-m4 (99-152 aa), diffused into the nucleus and cytoplasm because of their relatively small sizes (molecular mass $\leq 44.5 \mathrm{kDa}$ ), even if they did not contain a nonclassical NLS. By contrast, the mutant UL14-m3 (1-98 aa) showed exclusively nuclear staining, whereas UL14-m4 (99-152 aa) was diffuse both in the nucleus and cytoplasm. Therefore, we suggested that the nonclassical NLS was contained within the m3 segment.

Heat shock proteins (HSPs) are the most abundant intracellular proteins. HSP70 is a highly conserved member of the HSP family that primarily acts as a molecular chaperone, mediating functions such as nuclear transport [43, 44]. In HSV-1, the 44-104 aa of pUL14 can interact with VP16, and it shows homology to HSP70 [15]. To assess whether DEV pUL14 had similarities with HSV-1 pUL14, we conducted a BLAST search using the NCBI server. We found that DEV pUL14 had 46\% identity with HSV-1 and 
HSV-2 [12]. Regarding the differences between these proteins, ${ }_{60}$ RLKSR $_{64}$ of HSV-1 pUL14 was similar to that of HSP70. An $\alpha$-helix formed by this segment was integrated into the substrate-binding region [15]. In DEV, the sequence of pUL14 was ${ }_{60} \mathrm{QLRSN}_{64}$. The positively charged residues of $\mathrm{R}$ were replaced by the neutral residues $\mathrm{Q}$ and $\mathrm{N}$ at a critical position. This indicated that the characteristics of DEV pUL14 were different from those of HSV pUL14.

It has been investigated that NLS is a single peptide of basic amino acids [25-27]. Accordingly, only one cluster of basic residues ${ }_{8} R_{\text {RRRLR }}$ 13 was found in pUL14. To assess whether similarity existed between DEV pUL14 and HSP70, we conducted a BLAST search of their sequences. We found that the segment of ${ }_{8} R_{\text {RRRLR }}$, was similar to that of the ${ }_{25} \mathrm{AVRRLR}_{264}$ of HSP70. Moreover, this fragment, ${ }_{259} \mathrm{AVRRLR}_{264}$, is contained in the bipartite NLS of HSP70 [44-46]. Thus, we proposed that the cluster of basic residues, ${ }_{8} \mathrm{RQRRLR}_{13}$, played a key role like the NLS. The consensus sequence has been reported [47]. Furthermore, we found that the mutants could not localize to the nucleus unless mutants UL14-m1 (1-45 aa) and UL14-m2 (46$98 \mathrm{aa})$ recombined with each other. This finding indicated that the combined actions of the $2 \alpha$-helix fragments might account for the nuclear localization pattern. Additionally, there were $2 \alpha$-helixes in 1-98 aa (4-45 aa and 46-98 aa) that contained 34 and $26 \%$ basic amino acids, respectively. In 1-98 aa of pUL14, 9 protein-binding sites were identified. Thus, we suggested that the $2 \alpha$-helixes acted as a nonclassical NLS by forming a unique spatial conformation. However, it cannot be excluded that the deletion mutants disrupt the overall structure generated by the 2 proteins. It may also be likely that both proteins required a third unidentified partner to undergo relocalization.

The IFA and cotransfection assay results demonstrated that pUL14 interacted with VP16 in perinuclear regions, then both proteins entered into the nucleus during DEV assembly. It has been reported that tegument pro- tein, which is distributed in the nucleus, can be incorporated into the capsid during viral assembly [9]. In HSV-2, pUL14 has been established to interact with the capsid proteins pUL35 and pUL33, while VP16 contributed to capsid assembly [15]. In the present study, pUL14 and VP16 were both localized to the nucleus, suggesting that the 2 proteins might interact with capsid protein. Moreover, pUL14 and VP16 have been reported to be associated with glycoprotein, which indicates that a similar function might present in DEV during virion assembly. The time required for the relocalization of pUL14 and VP16 into the nucleus was $48-60 \mathrm{~h}$, indicating that relocalization occurred at a late stage. The first and secondary envelopment events occurred during this stage. The first envelopment occurred near the nuclear membrane. It has been previously reported that unenveloped capsids accumulated in the cytoplasm of UL14D-infected cells [17]. Moreover, the same authors suggested that pUL14 facilitated the addition of tegument and envelope for virion assembly. The findings of Yamauchi et al. [16] also indicated that pUL14 was required for the events that followed capsid formation. Those $2 \mathrm{DEV}$ proteins might associate with the mutation of tegument and envelope proteins, and this possibility merits further investigation.

In summary, the NLS was initially identified by constructing pUL14 truncation mutants. Moreover, pUL14 may be involved in the replication of viral DNA and assembly of both tegument and envelope.

\section{Acknowledgements}

This study was supported by grants from the National Natural Science Foundation of China (31272545), National Science and Technology Support Program (No. 2015BAD12B05), China Agricultural Research System (CARS-43-8), Special Fund for Key Laboratory of Animal Disease and Human Health of Sichuan Province (2016JPT0004), and Demonstration of Key Technologies for the Duck Industry in Sichuan Province (2014NZ0030).

\section{References}

1 Kaleta EF: Herpesviruses of birds - a review. Avian Pathol 1990;19:193-211.

2 Kaleta EF, Kuczka A, Kühnhold A, Bunzenthal C, Bönner BM, Hanka K, Redmann T, Yilmaz A: Outbreak of duck plague (duck herpesvirus enteritis) in numerous species of captive ducks and geese in temporal conjunction with enforced biosecurity (in-house keeping) due to the threat of avian influenza A virus of the subtype Asia H5N1. Dtsch Tierarztl Wochenschr 2007;114:3-11.
3 Woźniakowski G, Samorek-Salamonowicz E: First survey of the occurrence of duck enteritis virus (DEV) in free-ranging Polish water birds. Arch Virol 2007;159:1439-1444.

4 Metwally SA: Duck virus enteritis (duck plague); in Swayne DE, Glisson JR, McDougald LR, Nolan LK, Suarez DL, Nair V (eds): Diseases of Poultry, ed 13. Oxford, Blackwell Publishing, 2013, pp 431-439.

5 William WN, Jay CB: Time-dependent transformation of the herpesvirus tegument. J Virol 2009;83:8082-8089.
Identification of the NLS Region of DEV UL14 and Its Interaction with VP16
Intervirology 2016;59:187-196 DOI: $10.1159 / 000452711$ 
6 Tomtishen JP 3rd: Human cytomegalovirus tegument proteins (pp65, pp71, pp150, pp28). Virol J 2012;9:22.

7 Aggarwal A, Miranda-Saksena M, Boadle RA, Kelly BJ, Diefenbach RJ, Alam W, Cunningham AL: Ultrastructural visualization of individual tegument protein dissociation during entry of herpes simplex virus 1 into human and rat dorsal root ganglion neurons. J Virol 2012;86:6123-6137.

8 Owen DJ, Crump CM, Graham SC: Tegument assembly and secondary envelopment of Alphaherpesviruses. Viruses 2015;7:50845114.

9 Svobodova S, Bell S, Crump CM: Analysis of the interaction between the essential herpes simplex virus 1 tegument proteins VP16 and VP1/2. J Virol 2012;86:473-483.

10 Gross ST, Harley CA, Wilson DW: The cytoplasmic tail of herpes simplex virus glycoprotein $\mathrm{H}$ binds to the tegument protein VP16 in vitro and in vivo. Virology 2003;317:1-12.

11 Zhu Q, Courtney RJ: Chemical cross-linking of virion envelope and tegument protein of herpes simplex virus type 1 . Virology 1994; 204:590-599.

12 Ko DH, Cunningham AL, Diefenbach RJ: The major determinant for addition of tegument protein pUL48 (VP16) to capsids in herpes simplex virus type 1 is the presence of the major tegument protein pUL36 (VP1/2). J Virol 2009;84:1397-1405.

13 Schmelter J, Knez, J, Smiley JR, Capone JP: Identification and characterization of a small modular domain in the herpes simplex virus host shutoff protein sufficient for interaction with VP16. J Virol 1996;70:2124-2131.

14 Li YF, Huang B, Ma XL, Wu J, Li F, Ai W, Song MX, Yang HC: Molecular characterization of the genome of duck enteritis virus. Virology 2009;391:151-161.

15 Yamauchi Y, Wada K, Goshima F, Takakuwa H, Daikoku T, Yamada M, Nishiyama Y: The UL14 protein of herpes simplex virus type 2 translocates the minor capsid protein VP26 and the DNA cleavage and packaging UL33 protein into the nucleus of coexpressing cells. J Gen Virol 2001;82:321-330.

16 Yamauchi Y, Kiriyama K, Kubota N, Kimura H, Usukura J, Nishiyama Y, Nishiyama Y: The UL14 tegument protein of herpes simplex virus type 1 is required for efficient nuclear transport of the alpha transinducing factor VP16 and viral capsids. J Virol 2008;82:10941106.

17 Cunningham C, Davison AJ, MacLean AR, Taus NS, Baines JD: Herpes simplex virus type 1 gene UL14: phenotype of a null mutant and identification of the encoded protein. J Virol 2000;74:33-41.

18 Johnson DC, Baines JD: Herpesviruses remodel host membranes for virus egress. Nat Rev Microbiol 2011;9:382-394.

19 Elliott G, Mouzakitis G, O’Hare P: VP16 interacts via its activation domain with VP22, a tegument protein of herpes simplex virus, and is relocated to novel macromolecular assem- bly in coexpressing cells. J Virol 1995;69: 7932-7941.

20 Kamen DE, Gross ST, Girvin ME, Wilson DW: Structural basis for the physiological temperature dependence of the association of VP16 with the cytoplasmic tail of herpes simplex virus glycoprotein H. J Virol 2005;79: 6134-6141.

21 Nigg EA: Nucleocytoplasmic transport: signals, mechanisms and regulation. Nature 1997;386:779-787.

22 Yasuhara N, Oka M, Yoneda Y: The role of the nuclear transport system in cell differentiation. Semin Cell Dev Biol 2009;20:590-599.

23 Fagerlund R, Melen K, Kinnunen L, Julkunen I: Arginine/lysine-rich nuclear localization signals mediate interactions between dimeric STATs and importin alpha 5. J Biol Chem 2002;277:30072-30078.

24 Robbins J, Dilworth SM, Laskey RA, Dingwall C: Two interdependent basic domains in nucleoplasmin nuclear targeting sequence: identification of a class of bipartite nuclear targeting sequence. Cell 1991;64:615-623.

25 Lange A, McLane LM, Mills RE, Devine SE, Corbett AH: Expanding the definition of the classical bipartite nuclear localization signal. Traffic 2010;11:311-323.

26 Cervantes CF, Bergqvist S, Kjaergaard M, Kroon G, Sue SC, Dyson HJ, Komives EA: The RelA nuclear localization signal folds upon binding to IkBa. J Mol Biol 2011;405:754-764.

27 Zhou M, Liu HY, Xu XJ, Zhou H, Li XL, Peng C, Shen SR, Xiong W, Ma J, Zeng ZY, Fang SQ, Nie XM, Yang YX, Zhou J, Xiang JJ, Cao L, Li SF, Li GY: Identification of nuclear localization signal that governs nuclear import of BRD7 and its essential roles in inhibiting cell cycle progression. J Cell Biochem 2006;98: 920-930.

28 Yamauchi Y, Daikoku T, Goshima F, Nishiyama Y: Herpes simplex virus UL14 protein blocks apoptosis. Microbiol Immunol 2003; 47:685-689.

29 De Martino L, Marfe G, Di Stefano C, Paqnini U, Florio S, Crispino L, Iovane G, Macaluso $\mathrm{M}$, Giordano A: Interference of bovine herpesvirus 1 (BHV-1) in sorbitol-induced apoptosis. J Cell Biochem 2003;89:373-380.

30 Chkheidze AN, Liebhaber SA: A novel set of nuclear localization signals determine distributions of the $\alpha$-CP RNA-binding proteins. Mol Cell Biol 2003;23:8405-8415.

$31 \mathrm{Chu} \mathrm{CH}$, Chang LC, Hu HM, Wei SY, Liu HW, Lee Y, Kuo CC, Indra D, Chen C, Ong SJ, Tai JH: A highly organized structure mediating nuclear localization of a Myb2 transcription factor in the protozoan parasite trichomonas vaginalis. Eukaryot Cell 2011; 10:1607-1617.

32 Li FJ, Cheng AC, Wang MS: Bioinformatics analysis and characteristics of UL14 protein encoded by UL14 gene in duck enteritis virus. Adv Mater Res Vols 2012;669-675.

33 Guo YF, Shen CJ, Cheng AC, Wang MS, Zhang N, Chen S, Zhou Y: Anatid herpesvirus $1 \mathrm{CH}$ virulent strain induces syncytium and apoptosis in duck embryo fibroblast cultures. Vet Microbiol 2009;138:258-265.

$34 \mathrm{Wu}$ Y, Cheng AC, Wang MS, Yang Q, Zhu DK, Jia RY, Chen S, Zhou Y, Chen XY: Complete genomic sequence of Chinese virulent duck enteritis virus. J Virol 2012;86:5965.

35 Li LJ, Cheng AC, Wang MS, Xiang J, Zhang SC, Zhu DK, Jia RY, Luo QH, Zhou Y, Chen ZL, Chen XY: Expression and characterization of duck enteritis virus gI gene. Virol 2011;8:241.

36 Wada K, Goshima F, Takakuwa H, Yamada H, Daikoku T, Nishiyama Y: Identification and characterization of the UL14 gene product of herpes simplex virus type 2. J Gen Virol 1999;80:2423-2431.

$37 \mathrm{Hu}$ CD, Chinenov Y, Kerppola TK: Visualization of interactions among bZIP and Rel family protein in living cells using bimolecular fluorescence complementation. Mol Cell 2002;9:789-798.

38 Talaty P, Emety A, Everly DN: Characterization of the latent membrane protein 1 signaling complex of Epstein-Barr virus in the membrane of mammalian cells with bimolecular florescence complementation. Virol J 2011;8:414.

39 Kerppola TK: Design and implementation of bimolecular fluorescence complementation $(\mathrm{BiFC})$ assays for the visualization of protein interactions in living cells. Nat Protoc 2006; $1278-1286$

$40 \mathrm{He}$ Q, Cheng AC, Wang MS, Xiang J, Zhu DK, Zhou Y, Jia RY, Chen S, Chen ZL, Chen XY: Replication kinetics of duck enteritis virus UL16 gene in vitro. Virol J 2012;9:281.

41 Paine PL, Moore LC, Horowitz SB: Nuclear envelope permeability. Nature 1975;254:109-114.

42 Bizzarri R, Serresi M, Luin S, Beltram F: Green fluorescent protein based $\mathrm{pH}$ indicators for in vivo use: a review. Anal Bioanal Chem 2009; 393:1107-1122.

43 Kampinga HH, Craig EA: The HSP70 chaperone machinery: J proteins as drivers of functional specificity. Nat Rev Mol Cell Biol 2010; 11:579-592.

44 Fei J, Wang YS, Zhou Q, Gu JD: Cloning and expression analysis of HSP70 gene from mangrove plant Kandelia obovata under cold stress. Ecotoxicology 2015;24:1677-1685.

45 Wang KK, Shun Mei E, Jiang L, Zhang HL, Liu K, Zhang LL, Xiao XZ: Roles of nuclear localization signal (NLS) in inhibitory effect of HSP70 on nucleolar segregation induced by oxidative stress. Prog Biochem Biophys 2005;32:456-462.

46 Wang K, Deng G, Chen G, Liu M, Yi Y, Yang $\mathrm{T}$ : Heat shock protein 70 inhibits hydrogen peroxide-induced nucleolar fragmentation via suppressing cleavage and down-regulation of nucleolin. Cell Stress Chaperones 2012;17:121-130.

47 Dingwall C, Robbins J, Dilworth SM, Roberts $\mathrm{B}$, Richardson WD: The nucleoplasmin nuclear location sequence is larger and more complex than that of SV-40 large T antigen. J Cell Biol 1988;107:841-849. 